\title{
Avaliação de Aterosclerose em Transplantados Renais Através de Métodos Não Invasivos
}

\author{
Evaluation of Atherosclerosis in Renal Transplanted Patients by Non-Invasive Methods
}

\begin{abstract}
Cláudio Domênico Sahione Schettino ${ }^{1,2}$, Aristarco Gonçalves Siqueira Filho ${ }^{1}$, Romeu Cortes Domingues ${ }^{3}$, lugiro Kuroki ${ }^{3}$, Flávia Cristina Carvalho de Deus ${ }^{2,4}$, Renato Torres Gonçalves ${ }^{1}$, Hélio Magarino Torres ${ }^{5}$, Emerson Gasparetto ${ }^{1}$, Lúcio Cardoso Pacheco ${ }^{1}$, Eduardo Rocha ${ }^{1}$

Universidade Federal do Rio de Janeiro (UFRI)' , Clínica São Vicente², Clínica CDPI / Multimagem³, Instituto Nacional de Cardiologia4, Laboratório

Richet $^{5}$, Rio de Janeiro, RJ - Brasil
\end{abstract}

\section{Resumo}

Fundamento: A disfunção endotelial pode ser considerada um evento precoce da aterogênese.

Objetivo: Avaliar a aterosclerose em transplantados renais através do escore de cálcio coronariano, do duplex scan das carótidas e da reatividade braquial através do ultra-som.

Métodos: Avaliamos trinta transplantados renais do sexo masculino com função renal estável, idade média de 41,3 anos.

Resultados: A detecção da carga aterosclerótica nesta população foi significativa quando utilizada a técnica da reatividade braquial $(86,7 \%)$, menos freqüente baseando-se na presença de placa carotídea $(33,3 \%)$ ou no escore de cálcio coronariano (20\%). Placa carotídea foi considerada quando a espessura era superior a 12 mm. O escore de cálcio coronariano foi anormal quando acima de oitenta pela escala de Agatston, sendo observado em um percentual baixo $(21,7 \%)$ dos pacientes, possivelmente porque a tomografia pode não ser o método ideal para detectar aterosclerose em doentes renais, por não distinguir calcificações intimais da camada média. O controle clínico adequado, a baixa faixa etária e fatores relacionados ao tempo de diálise pré-transplante ou ao efeito antiinflamatório das drogas póstransplante podem retardar o aparecimento das calcificações.

Conclusão: A avaliação da carga aterosclerótica através do duplex scan das carótidas (33,3\%) e do escore de cálcio coronariano (20\%) não foi freqüente, não havendo correlação com o elevado índice de detecção de disfunção endotelial observado com o exame da reatividade braquial (86,7\%). (Arq Bras Cardiol 2009;92(5):369-374)

Palavras-chave: aterosclerose, transplantes/rim, endotélio/fisiopatologia.

\section{Summary}

Background: Endothelial dysfunction can be considered an early atherogenic event.

Objective: To assess atherosclerosis in renal transplanted patients through the coronary calcium score, carotid duplex scan and brachial reactivity through ultrasonography.

Methods: We assessed 30 renal transplanted male patients with stable renal function, with a mean age of 41.3 years.

Results: The detection of the atherosclerotic load in this population was very significant when the brachial reactivity technique was used (86.7\%); it was less frequent when based on the presence of carotid plaque (33.3\%) or the coronary calcium score (20\%). The carotid plaque was considered when the thickness was $>12 \mathrm{~mm}$. The coronary calcium score was abnormal when > 80 according to the Agatston scale, being observed in a low percentage of patients (21.7\%), possibly due to the fact that the tomography is not the ideal method to detect atherosclerosis in renal patients, as it does not differentiates intimal calcifications of the medial layer. The adequate clinical control, the low age range and the factors related to the time of pre-transplant dialysis or the anti-inflammatory effect of the post-transplant drugs can delay the onset of the calcifications.

Conclusion: The evaluation of the atherosclerotic load through the carotid duplex scan (33,3\%) and the coronary calcium score (20\%) was not frequent; there was no correlation with the high rate of endothelial dysfunction detection observed with the brachial reactivity assessment (86.7\%). (Arq Bras Cardiol 2009;92(5):339-344)

Key words: atherosclerosis; transplants/kidney; endothelium/phisiopathology.

Full texts in English - http://www.arquivosonline.com.br

Correspondência: Cláudio Domênico Sahione Schettino •

Rua Joana Angélica, 232, Ipanema, 22.420-030, Rio de Janeiro, RJ - Brasil

E-mail: cdomenico@gaveacor.com.br

Artigo recebido em 17/03/08; revisado recebido em 03/06/08; aceito em 03/06/08. 


\section{Introdução}

O endotélio vascular (EV) é uma barreira física que separa o sangue dos tecidos, capaz de liberar substâncias vasoconstrictoras e vasodilatadoras, mantendo a homeostase vascular ${ }^{1}$.

A disfunção endotelial (DE) pode ser entendida como um desequilíbrio entre os fatores que relaxam e que fazem a constrição do endotélio vascular. A DE pode ser vista como um evento precoce da aterogênese, sendo considerada fundamental na fisiopatologia dos eventos cardiovasculares e no processo aterosclerótico ${ }^{2}$. Vários exames podem ser utilizados, visando a avaliação da função endotelial (FE) e a detecção precoce de aterosclerose ${ }^{3}$.

A avaliação da aterosclerose deve ser iniciada pelo exame físico e complementada com exames especializados, tais como avaliação das artérias cervicais e periféricas e estudo do cálcio coronariano através da tomografia do tórax com multidetectores (MDCT) ${ }^{4}$.

A investigação da aterosclerose carotídea utiliza o duplex scan vascular a cores (DSC), buscando a detecção de placas carotídeas $^{5,6}$. A avaliação do escore de cálcio coronariano (ECC), realizado através da MDCT, é mais um método eficaz de investigação de doença cardiovascular em assintomáticos. O ECC normal é zero, e são estabelecidas quatro categorias de calcificação: 1 a $10=$ mínima, 11 a $100=$ leve, 101 a $400=$ moderada e acima de $400=$ importante. Finalmente, dentre os métodos de avaliação precoce da aterosclerose, destaca-se a técnica de avaliação da reatividade braquial (RB) por ultra-som. Este método foi descrito por Celemajer e cols. ${ }^{3}$, e baseia-se na capacidade dos vasos de responder a um estímulo mecânico, regular o seu tônus, e redistribuir o seu fluxo após cessação do estímulo, inferindo o grau de disfunção endotelial do vaso ${ }^{7}$.

Este exame não é invasivo ${ }^{3}$ e avalia o percentual de dilatação da artéria braquial, antes e após clampeamento com manguito aneróide de pressão. Quando este percentual é maior do que 10\% considera-se normal, ou seja, função endotelial preservada. Atualmente, existem diretrizes com normatizações da técnica ${ }^{7}$. Entretanto, poucos estudos investigaram de forma comparativa os diferentes métodos de avaliação da aterosclerose precoce.

O objetivo deste trabalho foi avaliar a carga aterosclerótica de transplantados renais através do ECC, do DSC das carótidas e da RB através do ultra-som.

\section{Métodos}

Foram selecionados aleatoriamente trinta transplantados renais do sexo masculino, todos com função renal estável, sendo sete receptores de rim de cadáver e os demais de doadores vivos. A idade média foi de 41,3 anos (variando entre 19 e 59 anos) e o índice de massa corpórea foi estimado entre 30 e 35 , em $53,3 \%$ dos pacientes, sendo que o restante tinha índice abaixo de 30 . Todos os participantes do estudo apresentavam mais de seis meses pós-transplante, para exclusão dos casos de rejeição aguda. A Tabela 1 resume os dados clínicos e laboratoriais da população estudada.
Todos os pacientes foram submetidos aos seguintes estudos: DSC de carótidas para verificação de placas carotídeas, MDCT de tórax para determinação do ECC e teste da RB para avaliação da FE.

O exame de DSC das carótidas foi realizado para avaliação da presença de placas $^{8}$, sendo considerada placa carotídea quando esta apresentava espessura acima de $12 \mathrm{~mm}$, tendo sido analisadas ambas as carótidas na máxima extensão possível.

A tomografia de tórax foi realizada em aparelho com quatro detectores, Somaton Plus Volume Zoom (Siemens, Alemanha), sendo o ponto de corte estabelecido como anormal quando acima de oitenta pela escala de Agaston, baseado no artigo de revisão sobre aterotrombose de Moreno e Furster ${ }^{9}$, que demonstrou que valores de ECC acima de oitenta, quando associados ao grupo intermediário pela escala de Framinghan, aumentavam a probabilidade pré-teste de mortalidade de 10 $\%$ em 10 anos para um valor de $27 \%$. Deve-se lembrar que este exame não utiliza nenhum tipo de contraste.

A técnica da RB utilizou o mesmo aparelho de ecocardiografia empregado para duplex scan de carótidas, além de manguitos para fazer a compressão da artéria, eletrodos e gel condutor de ultra-som, seguindo-se as orientações do Colégio Americano de Cardiologia7. Todos os pacientes estavam em jejum de pelo menos 8 horas. Em relação às considerações técnicas do exame, foi utilizada uma pressão de garroteamento $50 \mathrm{mmHg}$ acima da pressão arterial máxima, por um período de 5 minutos. A verificação da pressão arterial foi feita de forma manual. Apenas um caso tinha fístula artério-venosa funcionante, sendo examinado o braço contra-lateral onde não havia fístula. Não houve nenhuma complicação importante, a não ser dormência no braço examinado, queixa relatada por todos os indivíduos submetidos ao exame, sendo que esta desapareceu por completo após desinsuflação do manguito. Seis pacientes referiram cefaléia após uso do nitrato sublingual, e somente um indivíduo fez uso de dipirona para o alívio da cefaléia.

Todos os participantes do estudo assinaram termo de consentimento, sendo que sete pacientes recusaram-se a realizar o exame de tomografia para determinação do ECC. O estudo foi aprovado pelo Comitê de Ética da Universidade Federal do Rio de Janeiro. Para a análise estatística, foram utilizadas medidas descritivas para caracterização da amostra, tais como média, desvio-padrão, mínima e máxima. Na avaliação da disfunção endotelial, foi empregado o teste de Mc Nemar, sendo as diferenças estatisticamente significativas quando $\mathrm{p}<0,05$.

\section{Resultados}

A detecção da carga aterosclerótica nesta população de transplantados renais foi significativa quando utilizado a técnica da reatividade braquial $(86,7 \%)$, e menos freqüente baseando-se na presença de placa carotídea (33,3\%) ou no escore de cálcio coronariano (20\%).

A Tabela 2 demonstra que a média de aumento do diâmetro da artéria braquial, após a fase de vasodilatação fluxomediada, é baixa $(6,4 \%)$ denotando disfunção endotelial, sendo que em pacientes saudáveis são esperados valores acima de 10\%. 


\section{Artigo Original}

\section{Tabela 1 - Dados clínicos e laboratoriais de trinta pacientes transplantados renais}

\begin{tabular}{|c|c|c|c|c|c|c|c|}
\hline \multirow{2}{*}{ Variáveis } & \multicolumn{7}{|c|}{ Estatísticas descritivas } \\
\hline & Média & DP & Mínimo & $1^{0}$ quartil & Mediana & $3^{\circ}$ quartil & Máximo \\
\hline Idade (anos) & 41,3 & 10,1 & 19,0 & 35,5 & 41,0 & 49,3 & 59,0 \\
\hline $\mathrm{IMC}\left(\mathrm{kg} / \mathrm{m}^{2}\right)$ & 25,3 & 4,6 & 19,1 & 21,8 & 24,1 & 27,8 & 38,8 \\
\hline Glicose & 97,9 & 22,9 & 58,0 & 82,5 & 93,5 & 116,3 & 149,0 \\
\hline Uréia & 39,3 & 13,1 & 19,3 & 30,0 & 34,8 & 46,5 & 70,6 \\
\hline Cistatina C & 1,35 & 0,30 & 0,90 & 1,16 & 1,32 & 1,60 & 2,08 \\
\hline PCR-T & 8,06 & 26,26 & 0,13 & 0,68 & 1,67 & 4,28 & 144,20 \\
\hline Colesterol total & 191,9 & 45,6 & 125,0 & 165,5 & 183,0 & 210,3 & 367,0 \\
\hline Triglicerídeos & 182,4 & 104,6 & 68,0 & 124,0 & 147,0 & 224,8 & 528,0 \\
\hline HDL & 44,1 & 8,6 & 32,0 & 37,5 & 43,0 & 48,3 & 64,0 \\
\hline LDL & 110,3 & 31,1 & 49,0 & 89,8 & 106,5 & 131,0 & 197,0 \\
\hline Sódio & 140,2 & 2,0 & 137,0 & 138,8 & 140,0 & 141,3 & 145,0 \\
\hline Potássio & 4,42 & 0,40 & 3,70 & 4,10 & 4,35 & 4,70 & 5,70 \\
\hline Ácido úrico & 6,47 & 1,20 & 4,40 & 5,85 & 6,30 & 7,20 & 9,40 \\
\hline Clearance & 61,5 & 24,2 & 24,7 & 42,7 & 58,7 & 80,2 & 117,1 \\
\hline Hemáceas & 5,17 & 0,82 & 3,33 & 4,71 & 4,93 & 5,65 & 7,40 \\
\hline Hemoglobina & 14,8 & 2,1 & 9,9 & 13,5 & 14,2 & 15,9 & 21,0 \\
\hline Hematócrito & 44,9 & 6,4 & 31,5 & 41,6 & 43,3 & 47,9 & 63,9 \\
\hline Von Willebrand (\%) & 80,5 & 81,6 & 10,0 & 13,4 & 46,5 & 121,4 & 357,6 \\
\hline Pró-BNP & 314,1 & 453,6 & 10,6 & 55,7 & 148,4 & 292,0 & 2089,0 \\
\hline Interleucina 6 & 3,20 & 4,60 & 0,40 & 1,00 & 1,20 & 3,78 & 24,60 \\
\hline $\begin{array}{l}\text { Espessura da } \\
\text { intima }\end{array}$ & 0,70 & 0,21 & 0,40 & 0,50 & 0,60 & 0,90 & 1,10 \\
\hline Tamanho do rim & 11,7 & 1,0 & 9,3 & 11,0 & 11,8 & 12,4 & 13,4 \\
\hline Fluxo anast & 92,5 & 29,9 & 51,0 & 72,0 & 90,0 & 101,8 & 181,3 \\
\hline IR & 0,62 & 0,05 & 0,51 & 0,60 & 0,62 & 0,65 & 0,76 \\
\hline Cálcio & 77,0 & 179,8 & 0,0 & 0,0 & 0,0 & 28,6 & 750,0 \\
\hline $\begin{array}{l}\text { Pressão arterial } \\
\text { média }\end{array}$ & 107,0 & 20,0 & 73,3 & 93,3 & 106,7 & 117,5 & 153,3 \\
\hline
\end{tabular}

Tabela 2 - Estatísticas descritivas do diâmetro da artéria braquial e variação pós-isquemia em transplantados renais masculinos $(n=30)$

\begin{tabular}{lcccc}
\hline & Média & DP & Mínimo & Máximo \\
\hline $\begin{array}{l}\text { Diâmetro pré- } \\
\text { isquemia (mm) }\end{array}$ & 0,45 & 0,09 & 0,24 & 0,64 \\
\hline $\begin{array}{l}\text { Diâmetro pós- } \\
\text { isquemia (mm) }\end{array}$ & 0,47 & 0,09 & 0,27 & 0,67 \\
\hline Variação (\%) & 6,4 & 2,5 & 1,7 & 12,5 \\
\hline
\end{tabular}

Nota: variação percentual média para 22 "controles" saudáveis $=11,9 \%$.
O Gráfico 1 demonstra que a maioria dos pacientes estudados através de RB apresenta percentual de variação do diâmetro da artéria abaixo de $10 \%$.

O Gráfico 2 mostra a determinação da aterosclerose em transplantados renais, confrontando os métodos de RB, Placa Carotídea, ECC.

\section{Discussão}

Neste estudo, foi avaliada a carga aterosclerótica de pacientes transplantados renais através do escore de cálcio coronariano, do duplex scan das carótidas e da reatividade 


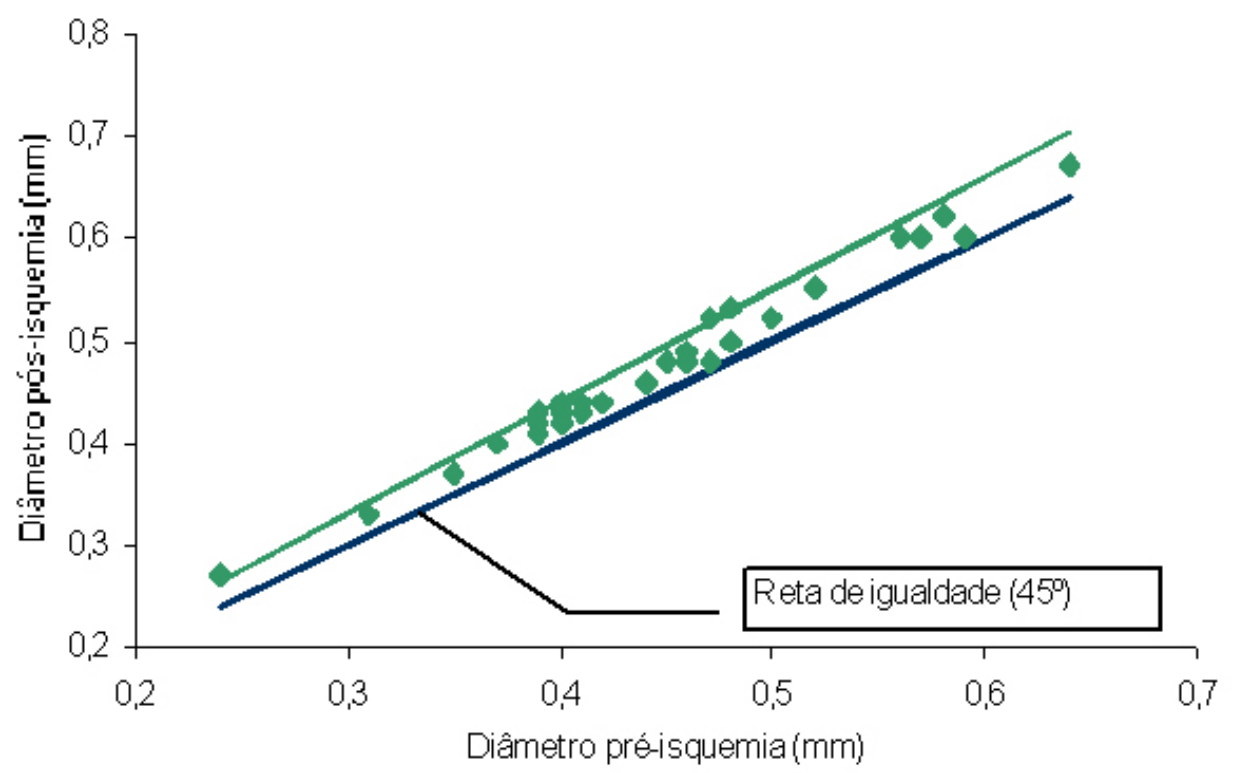

Gráfico 1 - Percentual de variação do diâmetro da artéria braquial através do exame de reatividade braquial.

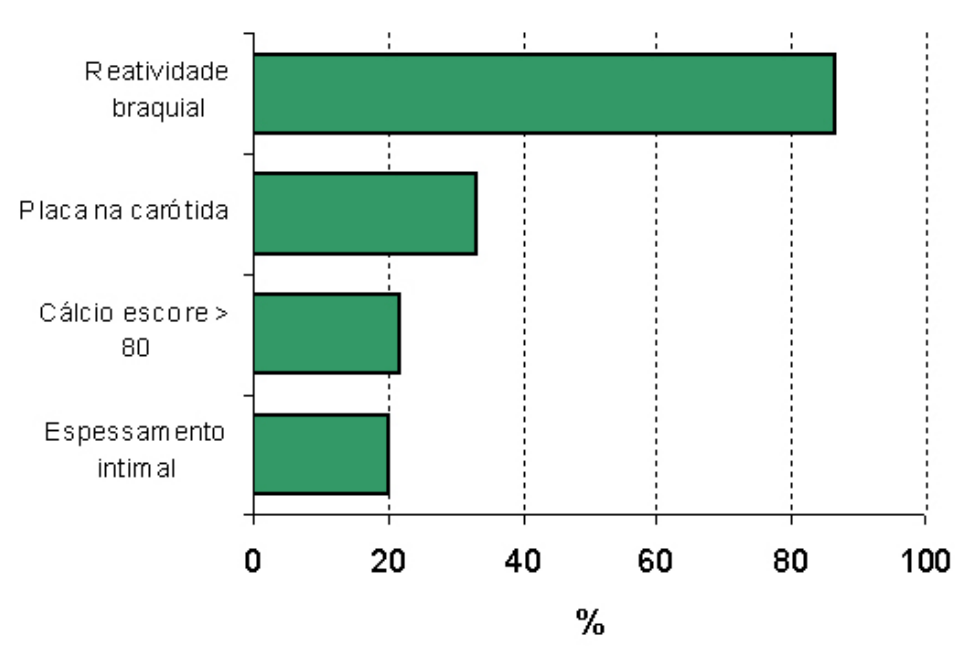

Gráfico 2 - Comparação da aterosclerose no grupo de pacientes estudados definida através de RB, Placa Carotídea, ECC e EIC (espessamento intimal carotídeo).

braquial através do ultra-som.

A aterosclerose é uma doença sistêmica, silente muitas vezes e potencialmente mortal, sendo que a redução de seu risco é custo-efetiva ${ }^{10}$. O que se faz necessário é a estratificação de forma criteriosa, permitindo identificar grupos de maior risco para a doença. O escore recomendado e utilizado habitualmente é o de Framinghan, que é bastante simples e de fácil aplicabilidade, mas que, no entanto, apresenta algumas limitações ${ }^{11,12}$. Na tentativa de se determinar os indivíduos considerados de médio risco, aqueles com probabilidade de $10 \%$, de ter um evento cardiovascular em dez anos, alguns testes vêm sendo utilizados, tais como, o DSC das carótidas, 
o ECC e os exames provocativos de isquemia. Cabe ressaltar que não existem ainda diretrizes estabelecidas para este grupo de risco intermediário ${ }^{13}$ e que a seleção dos exames deve ser criteriosa e individualizada, levando-se em consideração fatores de risco presentes e dados de história familiar.

Chambless e cols. ${ }^{14}$ demonstraram que tanto a redução dos níveis pressóricos e do colesterol, quanto o controle do tabagismo retardam a evolução da aterosclerose e da incidência de eventos coronarianos. O marcador ideal da aterosclerose deve ser sensível, de preferência não invasivo, deve ter relação com o desfecho de eventos cardiovasculares, com boa especificidade, e permitir diferenciar a presença ou ausência da doença cardiovascular ${ }^{15}$.

A determinação do espessamento intimal carotídeo está consolidada como um ótimo marcador na detecção da aterosclerose. Atualmente, o que se recomenda é que essa avaliação leve em conta a faixa etária, o sexo e a etnia, de modo que se possa estabelecer um paralelo entre a idade cronológica e a idade vascular ${ }^{16,17}$.

Não se sabe ainda qual é o melhor ponto de corte do ECC, mas valores $>400$ têm alta especificidade e correlação com lesão acima de $70 \%$ de obstrução coronariana. Newman e cols. ${ }^{4}$ avaliaram 414 indivíduos com idade média de 79 anos e observaram que $38 \%$ dos indivíduos tinham ECC > 400 . Além disso, demonstraram que $17 \%$ destes pacientes seriam considerados normais quando se utilizava uma composição de outros três métodos (ECG, índice tornozelo-braço e EIC). Mesmo em idades avançadas existe uma correlação entre calcificação coronarianos e infarto agudo do miocárdio ${ }^{18}$.

No presente estudo, o ECC foi considerado anormal quando $>80$ pela escala de Agatston, sendo observado em cinco $(21,7 \%)$ de 23 pacientes estudados. Este percentual é relativamente baixo, sendo que algumas possibilidades devem ser mencionadas. A primeira é que a tomografia pode não ser o método ideal para detectar aterosclerose em doentes renais devido à incapacidade de distinguir calcificações intimais da aterosclerose das calcificações da média, comuns em doentes renais ${ }^{19}$. Além disso, o controle clínico adequado dos pacientes estudados pode estar retardando o aparecimento das calcificações. Finalmente, a baixa faixa etária do grupo (média de 41,3 anos), a ausência de diabéticos, o bom controle medicamentoso da dislipidemia e da hipertensão arterial, associados ao curto período de tempo de diálise pré-transplante $(<1$ ano) e ao possível efeito antiinflamatório das drogas pós-transplante, podem estar retardando a calcificação coronarianos.

Sabe-se que a calcificação das artérias coronárias ocorre somente em artérias ateroescleróticas, e que a aterosclerose é necessária, mas não o suficiente para desenvolver um evento coronarianos. Nos doentes renais, algumas variáveis podem propiciar diferentes graus de calcificação coronariana, dentre as quais: o tempo de doença renal, a idade dos pacientes e a extensão da doença. Não se tem certeza se um desequilíbrio na relação de cálcio e fósforo, que pode estar presente nos doentes renais, seria também um fator a mais a favorecer um aumento no ECC destes pacientes ${ }^{20}$.

O exame da RB vem sendo utilizado mais como pesquisa do que como uma ferramenta clínica. Isto se deve a alguns fatores, dentre os quais: técnica nova ainda sendo validada, é um exame operador dependente e com diretrizes recentemente estabelecidas, e que vem sendo aprimorado. Sabe-se que a disfunção endotelial, que é avaliada pela técnica da reatividade braquial, pode preceder à formação de placas de ateroma e talvez ao dano na microcirculação, mas talvez não consiga quantificar o impacto do dano endotelial em outros órgãos ${ }^{21,22}$.

Uma das principais limitações deste estudo é que se trata de uma avaliação transversal. Ou seja, para avaliação da ateromatose subclínica, o tempo de exposição dos pacientes aos fatores de risco é uma dado importante. Talvez a faixa etária mais jovem da população estudada (média de 41 anos) represente uma segunda limitação. Em relação aos métodos utilizados, o espessamento intimal carotídeo e o escore de cálcio coronariano deveriam ser avaliados levandose em consideração faixa etária, sexo e até mesmo etnia. Infelizmente ainda não existem tabelas em nosso país com estes dados. Cabe ainda ressaltar que o exame da reatividade braquial é novo, operador dependente, e necessita de uma curva de aprendizado.

Deve-se destacar que a doença cardiovascular é a maior causa de mortalidade nos pacientes com insuficiência renal crônica avançada e naqueles submetidos a transplante renal ${ }^{23}$, sendo que a detecção de uma carga aterosclerótica importante pode ajudar na estratificação de risco desta população.

Concluindo, nesta casuística, a detecção da carga aterosclerótica foi mais significativa no exame de reatividade braquial e menos comum ao se avaliar a presença de placa carotídea ao duplex scan ou o escore de cálcio coronariano. Por se tratar de uma população de alto risco, transplantados renais talvez merecessem uma estratégia mais agressiva de investigação diagnóstica da função endotelial e de ateromatose subclínica incluindo exames de laboratório, teste da reatividade braquial, duplex scan de carótidas e avaliação do escore de cálcio coronariano. Novas estratégias para diminuir o risco de eventos cardiovasculares incluindo tratamentos individualizados e até mesmo mais intensos podem diminuir os eventos cardiovasculares ${ }^{24}$. Mais estudos serão necessários para confirmar se a detecção precoce de aterosclerose subclínica em populações de risco será beneficiada por uma estratégia de prevenção mais agressiva.

\section{Potencial Conflito de Interesses}

Declaro não haver conflito de interesses pertinentes.

\section{Fontes de Financiamento}

O presente estudo não teve fontes de financiamento externas.

\section{Vinculação Acadêmica}

Este artigo é parte de tese de Doutorado de Claudio Domenico Sahione Schettino pela Universidade Federal do Rio de Janeiro. 


\section{Referências}

1. Verma $S$, Anderson T. Fundamentals of endothelial function for the clinical cardiologist. Circulation. 2002; 105: 546-9.

2. Hermann J, Lerman A. The endothelium: dysfunction and beyond. J Nucl Cardiol. 2001; 8: 197-206.

3. Celermajer DS, Sorensen KE, Gooch VM, Spiegelhalter DJ, Miller OI, Sullivan ID, et al. Non invasive detection of endothelial dysfunction in children and adults at risk of atherosclerosis. Lancet. 1992; 30: 1111-5.

4. Newman AB, Naydeck BL, Sutton-Tyrrell K, Edmundowicz D, leary DO, Kronmal R, et al. Relationship between coronary artery calcification and other measures of subclinical cardiovascular disease in older adults. Arterioscler Thromb Vasc Biol. 2002; 22: 1674-9.

5. Ebrahim S, Papacosta O, Whincup P, Wannamethee G, Walker M, Nicolaides AN, et al. Carotid plaque, intima media thickness, cardiovascular risk factors, and prevalent cardiovascular disease in men and women. The British Regional Heart Study. Stroke. 1999; 30: 841-50.

6. Held C, Hjemdahl P, Eriksson SV, Bjorkander I, Forslund L, Rehnqvist N. Prognostic implications of intima media thickness and plaques in the carotid and femoral arteries in patients with stable angina pectoris. Eur Heart J. 2001; 22: $62-72$.

7. Corretti MC, Anderson TJ, Benjamim EJ, Celermajer D, Charbonneau F, Creager MA, et al. Guidelines for the ultrasound assessment of the endothelial-dependent flow mediated vasodilation of the brachial artery: a report of the International Brachial Artery Reactivity Task Force. J Am Col Cardiol. 2002; 39: 257-65.

8. Douglas PS. Using imaging for cardiovascular risk prediction: an overview. J Am Soc Echocardiogr. 2004; 17: 350-2.

9. Moreno PR, Fuster V. The year in atherothrombosis. J Am Coll Cardiol. 2004; 44: 2099-110.

10. Cohn JN, Quyyuni AA, Hollenberg NK. Surrogate markers for cardiovascular disease: functional markers. Circulation. 2004; 110 (Suppl. 4): IV31-IV36.

11. Wilson P W, D'Agostinho R B, Levy D Prediction of coronary heart disease using risk factor categories. Circulation. 1998; 97: 1837-45.

12. D`Agostinho RB Sr, Grundy S, Sullivan LM, Wilson P, CDH Risk Prediction Risk. Validation of the Framingham coronary heart disease prediction scores: results of a multiple ethnic groups investigation. JAMA. 2001; 286 (2): 180-7.
13. Greeland P, Gaziano M Selecting asymptomatic patients for coronary computed tomography or electrocardiography exercise testing. N Engl J Med. 2003; 349: 465-73.

14. Chambless LE, Folson AR, Davis V, Sharrett R, Heiss G, Sorlie P, et al. Risk factors for progression of common carotid atherosclerosis: the Atherosclerosis Risk in Communities Study, 1987-1998. Am J Epidemiol. 2002; 155: 3847.

15. de Groot E, Hovingh K, Wiegman A, Duriez P, Smit AJ, Fruchart JC, et al. Measurement of arterial wall thickness as a surrogate marker for atherosclerosis. Circulation. 2004; 109 (23 Suppl. 1): III33-8.

16. Stein JH, Douglas PS, Srinivasan SR, Bond MG, Tang R, Li S, et al Distribution and cross sectional age related increases of carotid artery intima media thickness in young adults: the Bogalusa Heart Study. Stroke. $2004 ; 35: 2782-7$

17. Stein JH. Carotid intima media thickness and vascular age: you are only as old your arteries look. J Am Soc Echocardiogr. 2004; 17: 686-9.

18. Thompson GR, Partridge J. Coronary calcification score: the coronary-risk impact factor. Lancet. 2004; 363: 557-9.

19. Sarnak MJ, Levey AS, Schoolwerth AC, Coresh J, Culleton B, Hamm LL, et al. AHA Councils on Kidney in Cardiovascular Disease. High Blood Pressure Research, Clinical Cardiology, and Epidemiology and Prevention. Circulation. 2003; 108: 2154-69

20. McCullough PA, Soman S. Cardiovascular calcification in patients with chronic renal failure: are we on target with this risk factor? Kidney Int Suppl. 2004; 90: 518-24

21. Kuvin JT, Karas RH. Clinical utility of endothelial function testing. Circulation. 2003; 107: 3243-7.

22. Vogel RA, Correti MC, Plotnick GD. A comparision of braquial artery flow - mediated vasodilatation using upper and lower arm arterial occlusion in subjects with and without coronary risk factors. Clin Cardiol. 2000; 23: 571-5.

23. de Souza Delgado D, Gerola LR, Hossne NA Jr, Branco JN, Buffolo E. Revascularização do miocárdio em pacientes com transplante renal. Arq Bras Cardiol. 2002; 79 (5): 476-9.

24. Gill JS. Cardiovascular disease in transplant recipients: current and future strategies. Clin J Am Soc Nephrol. 2008; 3 (Suppl 2): S29-37. 